\section{DIAGNOSIS OF LEFT VENTRICULAR DIASTOLIC DYSFUNCTION ON RESTING ECG - A PROSPECTIVE STUDY}

KEY WORDS: Diastolic

Dysfunction, Resting ECG, Morris Index, corrected QT Interval

\title{
Sharada Sivaram Kalavakolanu*
}

\section{Mohana Priya}

\section{Associate Professor, Department of Cardiology. *Corresponding Author}

Background: Heart failure due to preserved as well as reduced ejection fraction is a major healthcare challenge; echocardiography, including tissue Doppler Imaging (TDI) serves as the cornerstone of its diagnosis. ECG is a widely available, relatively inexpensive tool for predicting left ventricular diastolic dysfunction (LVDD).

Objectives: We aimed to study different parameters in resting 12-lead ECG to evaluate whether they can predict LVDD diagnosed on detailed echocardiography including TDI.

Methods: This was a cross-sectional study of 39 patients undergoing echocardiographic study in cardiology department of our institute. They were classified into two groups based on LVDD (Group 1 Normal, Group 2 LVDD) and their resting 12-lead ECG was analyzed for following parameters: QT interval, corrected QT interval, $\mathrm{P}$ wave duration (PW-D) and height (PW-H) in Lead 2, P terminal wave duration ( $\mathrm{P}$ term-D) and height ( $\mathrm{P}$ term-H) in lead Vl, $\mathrm{P}$ wave terminal force in leadVl. Correlation of these parameters with LVDD was investigated.

Results: Study included 39 patients, females 16, males 23, with age range 20-87 (mean 48) years. Group 1 comprised of 14 normal echo patients and 25 patients with LVDD were in Group 2. The Mean QTc was $419 \mathrm{~ms}$ (SD 21) and 440ms (SD 30) in Group 1 and Group 2 respectively, which was statistically significant $(p=0.03)$. The $P$ terminal wave duration V1 difference was statistically significant $(p 0.03$ ) with a mean of $39 \pm 17 \mathrm{~ms}$ vs $52 \pm 19 \mathrm{~ms}$ in Grp 1 and Grp 2 respectively. $P$ terminal wave height $\mathrm{Vl}$ was $0.79 \pm 0.26 \mathrm{~mm}$ in Grp 1 and $1.1 \pm 0.41 \mathrm{~mm}$ in Grp 2, which was statistically significant ( $p=$ $0.01)$. Morris Index was statistically highly significant between the two groups $(0.03 \pm 0.02 \mathrm{~mm} . \mathrm{s}$ Grpl and $0.07 \pm 0.04$ mm.s in Grp2, p 0.009). P wave height lead 2 was $1.1 \pm 0.4 \mathrm{~mm}$ and $1.7 \pm 0.6 \mathrm{~mm}$ in Grp 1 and Grp 2 respectively $(p=0.002)$. $P$ wave duration in Lead 2 did not find significance statistically ( $p 0.08$ ) with mean $90 \pm 19 \mathrm{~ms}$ versus $103 \pm 26 \mathrm{~ms}$ in Group 1 and 2 respectively.

Conclusion: QTc, P terminal wave duration and amplitude in Vl, Morris Index, and P wave amplitude in lead 2 are significantly higher in patients with echocardiographic diagnosis of LVDD.

\section{INTRODUCTION}

Left ventricular diastolic dysfunction (LVDD) is defined as "inability of the LV to fill during rest or exercise, to a normal end-diastolic volume without an abnormal increase in LV end diastolic pressure" '. Prevalence of asymptomatic diastolic dysfunction in the general community is $\sim 25-30 \%$ in individuals $\geq 45$ years ${ }^{2}$, while diastolic function is frequently abnormal in patients with systolic dysfunction.

Diagnostic evidence of LVDD can be obtained invasively (as elevated LV end-diastolic pressure) or non-invasively by TDI, coupled with plasma levels of natriuretic peptide ${ }^{3}$. Several echocardiographic parameters, such as, blood flow Doppler of mitral valve or pulmonary veins, echo measures of LV mass index or left atrial volume index have been validated to diagnose LVDD ${ }^{3}$.

ECG is a widely available, even in primary care, unlike echocardiography. Hence, there has been interest in investigating ECG features to predict $\operatorname{LVDD}^{4,5}$. QTc interval prolongation has shown to be predictive and correlate with LVDD in previous studies ${ }^{4,5}$. ECG features of left atrial dilatation, such as prolonged $\mathrm{P}$ wave duration, increased $\mathrm{P}$ wave dispersion, have specifically been focus of interest and have shown to correlate well with LVDD.

\section{AIM OF THE STUDY}

In this study, we aimed to study different parameters in resting 12-lead ECG and evaluate whether they can predict left ventricular diastolic dysfunction (LVDD) diagnosed by detailed echocardiography including Tissue Doppler Imaging (TDI).

\section{SUBJECTS AND METHODS}

This cross-sectional study was carried out in the outpatient department (OPD) of cardiology, at our institute. Consecutive patients who attended cardiology OPD and underwent ECG and detailed echo including TDI were included in the study.

\section{Inclusion Criteria:}

A) age above 18 years

B) clinically stable

\section{Exclusion Criteria:}

A) active ischemia

B) dyselectrolytemia

C) Were on QT prolonging drugs/ anti-arrhythmic (class 1 and class 3 ) drugs.

D) Presence of atrial flutter/ fibrillation, bundle branch block or Wolff-Parkinson-White syndrome on baseline ECG.

\section{ECG Measurements:}

These were performed with digital calipers on desktop screen image of the 12-lead ECG.

1. QT interval and corrected QT interval (QTc) ms: This was measured from the beginning of longest QRS complex to the end of T wave. QTc was obtained by dividing the actual QT by the square root of RR interval.

2. $\mathrm{P}$ wave duration in lead 2 (PWD-L2) ms: In L2, $\mathrm{P}$ wave duration was measured beginning to end as it crosses isoelectric line in 3 beats and average of the 3 beats was taken.

3. P wave height amplitude in lead 2 (PWH-L2) mm: TP segment was considered isoelectric and in lead $2, \mathrm{P}$ wave height was measured from its peak to isoelectric line.

4. P terminal wave duration in lead Vl (P TermD-Vl) ms: Measured as duration of downward deflection of terminal part of $P$ wave in leadVl

5. $\mathrm{P}$ terminal wave height in lead Vl (P TermH-Vl) $\mathrm{mm}$ : Measured as amplitude of downward deflection of terminal part of $P$ wave in leadVl.

6. Morris Index: $\mathrm{P}$ wave terminal force in lead Vl (PTFVl), referred to as the Morris index, is a product of the amplitude $(\mathrm{mm})$ and time $(\mathrm{s})$ of a terminal negative $P$ wave6. Normal value is $<0.04 \mathrm{~mm} \cdot \mathrm{s}$. 


\section{Echocardiographic measurements}

Trans-thoracic imaging was performed in the left lateral decubitus position using a GEVivid T9 machine (GE Medical Systems, WI), equipped with $2.5 \mathrm{MHz}$ phase array transducer and TDI software. A detailed echocardiographic study using conventional and TDI techniques was done on all patients to estimate diastolic LV function5. The ratio of trans-mitral early LV filling velocity $(E)$ to early diastolic Doppler tissue imaging velocity of the mitral annulus (transmitral E/E') was computed and if $\geq 15$ was considered abnormal and a reliable indicator of LVDD(ESC). Patients were classified into 2 groups based on this ratio- those with normal E/E' in Group 1 and those with abnormal value into Group 2

\section{Statistical Analysis:}

Statistical analysis was performed using IBM SPSS statistical software, version 21.0 (SPSS, Chicago, IL). Values for continuous variables are expressed as mean $\pm \mathrm{SD}$, whereas, for categorical variables, as observed number and percentage. Independent $t$ test was used for comparisons between the 2 patient groups, for continuous variables. Significance level was set at $\mathrm{p} \leq .05$.

\section{RESULTS}

Study included 39 patients, males 23 and females 16, with age range 20-87 (mean 48) years. Group 1 comprised of 14 patients with no LVDD on echo and Group 2 consisted of 25 patients with LVDD. In 5 patients of Grp 2 , there was additional significant $\mathrm{LV}$ systolic dysfunction.

The Mean QTc was 419ms (SD 21) and 440ms (SD 30) in Group 1 and Group 2 respectively, which was statistically significant $(p=0.03)$. The $P$ terminal wave duration $\mathrm{Vl}$ difference was statistically significant ( $p 0.03$ ) with a mean of $39 \pm 17 \mathrm{~ms}$ vs $52 \pm 19 \mathrm{~ms}$ in Grp 1 and Grp 2 respectively. $P$ terminal wave height Vl was $31 \pm 10 \mathrm{~ms}$ in Grp 1 and $44 \pm 16 \mathrm{~ms}$ in Grp 2, which was statistically significant $(p=0.01)$. $P$ wave height lead 2 was $44 \pm 16 \mathrm{~ms}$ an $69 \pm 25 \mathrm{~ms}$ in Grp 1 and Grp 2 respectively $(\mathrm{p}=$ 0.002 ). $P$ wave duration in Lead 2 did not find significance statistically ( $\mathrm{p} 0.08$ ) with mean $90 \pm 19 \mathrm{~ms}$ versus $103 \pm 26 \mathrm{~ms}$ in Group 1 and 2 respectively.(Table 1 ).

Table - 1 Comparison Of Ecg Parameters In Patients With Nrmal (group 1) And Impaired Lv Diastolic Function (group 2).

\begin{tabular}{|l|l|l|l|}
\hline ECG Variable & Group 1 (n 14) & Group 2 (n 25) & $\begin{array}{l}\text { p value (Sig } \\
\leq 0.05)\end{array}$ \\
\hline QTc ms & $419 \pm 21$ & $440 \pm 30$ & 0.028 \\
\hline $\begin{array}{l}\text { P Term D-Vl } \\
\text { ms }\end{array}$ & $39 \pm 18$ & $53 \pm 19$ & 0.032 \\
\hline $\begin{array}{l}\text { P Term H-Vl } \\
\text { mm }\end{array}$ & $0.79 \pm 0.26$ & $1.1 \pm 0.41$ & 0.010 \\
\hline Pterm F-Vl & $0.03 \pm 0.02$ & $0.07 \pm 0.04$ & 0.009 \\
\hline PW H-L2 mm & $1.1 \pm 0.4$ & $1.7 \pm 0.6$ & 0.002 \\
\hline PW D-L2 ms & $90 \pm 19$ & $103 \pm 26$ & 0.08 \\
\hline
\end{tabular}

OTc: corrected $Q T$ interval, PTermD-Vl: $P$ terminal wave duration Vl. PTerm H-Vl: P terminal wave height Vl, PTerm FV1: $P$ terminal Force V1, PW H-L2: $P$ wave height lead2, PW D-L2: P wave duration L2.

\section{DISCUSSION}

In this prospective study of 39 consecutive patients, we found several ECG parameters to be of significantly higher occurrence in patients with LVDD. These included corrected QT interval, P terminal wave duration and height in lead V1,Morris Index, $\mathrm{P}$ wave height in lead 2. However, the $\mathrm{P}$ wave duration in lead 2 did not attain but showed trend towards statistical significance, probably due to small sample size.

\section{Qtc And Qt Interval ${ }^{4,5,7}$ :}

We found mean QTc was significantly more prolonged in LVDD than in normal subjects, similar to other studies. In studies of individuals without CAD (based on stress ECG or
CAG), QT and QTc intervals were significantly longer in individuals with LVDD compared to individuals without LVDD $^{5}$. QTc interval $\geq 435 \mathrm{~ms}$ had a sensitivity and specificity of 73 and $74 \%$. Similar results were reported in patients with the suspicion of heart failure 4 . QTc prolongation can be attributed to many factors: altered intracellular calcium handling in cardiomyocytes, autonomic imbalance, or influences of comorbidities.

\section{P Terminal Wave Duration And Height In Lead Vl And Morris Index:}

$\mathrm{P}$ terminal wave duration as well as height were found to be significantly greater in LVDD in our study, similar to other studies $(5,7)$. In left atrial enlargement, duration of terminal negative $P$ wave deflection in leadVI $\geq 0.04 \mathrm{sec}$ and amplitude of terminal negative $P$ wave deflection in leadVI $\geq 0.10 \mathrm{mV}$.

Morris Index or P-wave terminal force in lead Vl (PTF-VI) is product of $\mathrm{P}$ terminal wave duration and amplitude in lead Vlnormal value being $<0.01 \mathrm{~mm}$.s, in LAE it is $\geq 0.04 \mathrm{~mm}$.s. PTF$\mathrm{Vl}$ is an ECG marker of increased LA volume, elevated $\mathrm{LA}$ filling pressures and unfavorable $\mathrm{LA}$ remodeling as a consequence of atrial contraction against a stiffened LV. LIFE study found abnormal $\mathrm{P}$-wave terminal force in lead Vl to be associated with worse diastolic function. Abnormal PTF-Vl values are related to left ventricular diastolic dysfunction, atrial fibrillation, congestive heart failure, stroke, and sudden death(8).

\section{P-Wave Amplitude Lead2:}

We found higher $\mathrm{P}$ wave amplitude in lead 2 in LVDD. Normal $P$ wave amplitude in $\mathrm{L} 2$ is $<2.5 \mathrm{~mm}$ or $<0.25 \mathrm{mv}$. Hayiroglu et al measured amplitude of $\mathrm{P}$-wave with LVDD as an outcome in 204 individuals without CAD or other major cardiac pathologies visiting the outpatient cardiology clinic ${ }^{9}$. At a cutoff value of $0.102 \mathrm{mV}$, this parameter showed a sensitivity of $67 \%$ and specificity of $60 \%$.

\section{P wave duration Lead 2:}

In our study, $\mathrm{P}$ wave duration in L2 was not significantly different between the groups ( $p$ 0.08), probably due to small sample size. In a study by Sumita et al, P-wave duration $>$ $110 \mathrm{~ms}$ was more sensitive for LVDD (sensitivity $86 \%$, specificity $86 \%$ ), and a P-wave duration> $120 \mathrm{~ms}$ was more specific for LVDD (sensitivity $34 \%$ and specificity $100 \%$ ) $^{10}$.

\section{Strength and Limitations of our study:}

Confirms reliability of abnormal $\mathrm{P}$ terminal force in lead Vl and utility of prolonged QTC in the diagnosis of LVDD. Limitations were small sample size and no distinction was made to account for gender in analyzing ECG variables, known to be gender-dependent (such as QT interval).

\section{CONCLUSIONS}

ECG is an widely available, relatively inexpensive investigation in primary care setting. Resting ECG parameters such as corrected QT interval, Morris Index, P terminal wave duration and amplitude in lead Vl and $\mathrm{P}$ wave amplitude in lead 2 were found to be significantly higher in patients with LV diastolic dysfunction. Hence, their identification helps in stratification of these patients for further investigations with a specialist, including echocardiogram with Tissue Doppler Imaging.

Acknowledgement: The statistical analysis provided by Ms.Sujini Dharani, lecturer \& statistician in our institute (MMCHRI) is gratefully acknowledged.

\section{REFERENCES:}

[1] Appleton CP, Firstenberg MS, Garcia MJ, Thomas JD. The echo-Doppler evaluation of left ventricular diastolic function. A current perspective. Cardiol Clin. 2000 Aug;18(3):513-46,ix.

[2] Abhayaratna WP, Marwick TH, Smith WT, Becker NG. Characteristics of left ventricular diastolic dysfunction in the community: an echocardiographic survey. Heart. 2006 Sep;92(9):1259-64.

[3] Paulus WJ, Tschöpe C, Sanderson JE, et al. How to diagnose diastolic heart 
failure: a consensus statement on the diagnosis of heart failure with normal left ventricular ejection fraction by the Heart Failure and Echocardiography Associations of the European Society of Cardiology. Eur Heart J. 2007;28:2539-50.

[4] Van Ommen AM, Kessler EL, Valstar G, et al. Electrocardiographic Features of Left Ventricular Diastolic Dysfunction and Heart Failure With Preserved Ejection Fraction:A Systematic Review.Front Cardiovasc Med.2021;8:772803.

[5] Taha T, Sayed K, Saad M and Samir M. How accurate can electrocardiogram predict left ventricular diastolic dysfunction? Egyptian HeartJ.2016;68:117-123.

[6] Lin H, Lin T, Lin L, Ye M. Roles of Morris Index on Poor Outcomes in Patients with Non-ST Segment Elevation Acute Coronary Syndrome. Med Sci Monit. 2020;26:e924418.

[7] Wilcox JE, Rosenberg J, Vallakati A, et al. Usefulness of electrocardiographic QT interval to predict left ventricular diastolic dysfunction. Am J Cardiol. 2011;108:1760-6.

[8] Tanoue MT,Kjeldsen SE,Devereux RB \& Okin PM.Relationship between abnormal P-wave terminal force in lead Vl and left ventricular diastolic dysfunction in hypertensive patients: the LIFE study.Blood Pressure.2017;26:94-101.

[9] Hayiroglu MI, Çinar T, Çiçek V, et al. A simple formula to predict echocardiographic diastolic dysfunction-electrocardiographic diastolic index. Herz.2021;46:159-65.

[10] Sumita Y, Nakatani S, Murakami I, Taniguchi M. Significance of left atrial overload by electrocardiogram in the assessment of left ventricular diastolic dysfunction.J Echocardiogr. 2019; 18:105-12. 